\title{
Der ischämische Schlaganfall
}

\author{
Andreas Unterberg
}

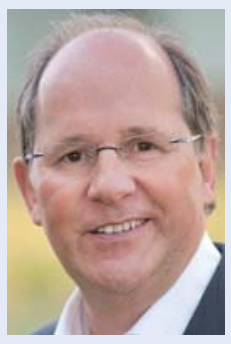

D

er ischämische Schlaganfall ist eine Erkran-

kung, die häufig desaströse Folgen für den

Patienten hat. So ist der Schlaganfall oft

mit dauernder, erheblicher körperlicher

Behinderung vergesellschaftet und somit ein großes medizinisches und sozioökonomisches Problem.

Ohne jeden Zweifel sind in den letzten Jahrzehnten erhebliche Fortschritte in der Behandlung des ischämischen Schlaganfalls erzielt und umgesetzt worden. Besonders ist in diesem Zusammenhang auf die intravenöse Lyse mit rtPA hinzuweisen. Aber auch die Einführung von flächendeckenden Stroke-Units mit einer konsequenten frühzeitigen Diagnostik und Normalisierung des „Milieu interne“ haben signifikante Fortschritte gezeitigt. Darüber hinaus ist die Prophylaxe weiterer akut ischämischer Läsionen von großer Bedeutung.

Dennoch gibt es nach wie vor ein erhebliches Potenzial zur weiteren Verbesserung der Behandlung. Dabei spielt die Wiedereröffnung von verschlossenen Gefäßen eine Schlüsselrolle. Dies ist bereits seit mindestens zwei Dekaden erkannt, aber ein schwer zu erreichendes Ziel.

Zwei Dinge sind bei der Rekanalisation bedeutsam: Effizienz und Schnelligkeit.

Die Schnelligkeit, ein verschlossenes Gefäß wieder zu eröffnen, hängt in erster Linie davon ab, wie rasch betroffene Patienten in ärztliche Behandlung gelangen - und hier natürlich auch in Zentren, in denen eine adäquate Behandlung möglich ist. Und wie gelingt es, verschlossene zerebrale Gefäße wieder zu eröffnen?

\section{Korrespondenzadresse}

Prof. Dr. med.

Andreas Unterberg

Universitätsklinikum

Heidelberg

Neurochirurgische Klinik

Im Neuenheimer Feld 400

69120 Heidelberg

E-Mail: andreas.unterberg

@med.uni-heidelberg.de
Die Antwort darauf gibt der exzellente Übersichtsartikel von Rohde, Jansen und Bendszus mit dem Titel Neuro-Thrombektomie - Mechanische Rekanalisierung akuter zerebraler Gefäßverschlüsse hier in dieser Ausgabe.

Es werden die unterschiedlichen technischen und klinischen Aspekte der mechanischen Rekanalisation bei akuten zerebralen Gefäßverschlüssen dargestellt.
Die Entwicklung dieser Techniken begann in den 90erJahren. Zwar wurde rasch in der PROACT II-Studie mit intraarterieller Lyse ein eigentlich positives Resultat erzielt, doch fragt man sich, warum letztlich weder in den USA noch in Europa eine Zulassung für das Verfahren erfolgte.

Positiv an dieser Nichtzulassung war möglicherweise die Weiterentwicklung anderer Rekanalisationsmethoden, wie die Entwicklung intrakranieller Stents oder die Entwicklung von „Stent-Retrievern“. Durch beide Verfahren wurde die Rekanalisationsrate noch einmal besser. Allerdings sind beide Verfahren bisher nicht „Standard“. Warum?

Bis heute fehlt es bedauerlicherweise an überzeugenden klinischen Resultaten. Offenbar sind interventionell tätige Radiologen wie Chirurgen oft verliebt in ihre Techniken und technische Resultate. Leider ist jedoch ein technisches Resultat, in diesem Fall die Rekanalisationsrate, nicht das letzte Maß, sondern die messbare klinisch-neurologische Besserung oder aber die messbare Reduktion eines Schadens.

Es ist den Autoren der Übersichtsarbeit sehr wohl bewusst, dass dies derzeit der „Knackpunkt“ der Neurothrombektomie ist. Effektivität und Sicherheit sind bereits überzeugend gezeigt. Es fehlt der Nachweis der klinischen Effektivität. Und an dieser Stelle kommt wieder der bereits oben angeführte Zeitfaktor ins Spiel. Nach wie vor wissen wir nicht, wie lang das Zeitfenster für eine im Hinblick auf das klinische Outcome effektive Rekanalisation offen ist, geschweige denn, wie man dieses Fenster möglicherweise längere Zeit offenhalten kann.

Schlussendlich: Sobald gezeigt werden kann, dass die Rekanalisation durch Neurothrombektomie die bleibende Behinderung nach einem ischämischen Schlaganfall signifikant reduziert, ist der Weg frei für eine Umsetzung in der Praxis und für die Etablierung neuer Standards. Ich bin davon überzeugt, dass dann auch neurovaskuläre Zentren eine Zukunft besitzen, und ich bin optimistisch, dass dies in naher Zukunft passiert. Die entsprechenden Studien laufen bereits. 